\title{
A splicing variation in NPRL2 causing familial focal epilepsy with variable foci: additional cases and literature review
}

\author{
Jia Zhang ${ }^{1,2}$, Yajun Shen ${ }^{1,2}$, Zuozhen Yang ${ }^{3}$, Fan Yang ${ }^{3}$, Yang $\mathrm{Li}^{1,2}$, Bo Yu ${ }^{4}$, Wanlin Chen ${ }^{4}$ and Jing Gan ${ }^{1,2}$
}

(c) The Author(s) 2021

NPRL2 (nitrogen permease regulator like 2) is a component of the GATOR1(GAP activity towards rags complex 1) proteins, which is an inhibitor of the amino acid-sensing branch of the mTORC1 pathway. GATOR1 complex variations were reported to correlate with familial focal epilepsy with variable foci (FFEVF). However, FFEVF caused by NPRL2 variants has not been widely explored. Here, we describe a variant, $339+2 T>C$, in NPRL2 identified by trio whole-exome sequencing (WES) in a family. This splicing variant that occurred at the $5^{\prime}$ end of exon 3 was confirmed by minigene assays, which affected alternative splicing and led to exon 3 skipping in NPRL2. Our cases presented multiple seizure types (febrile seizures, infantile spasms, focal seizures, or focal to generalized tonic-clonic seizures). Electroencephalogram (EEG) showed frequent discharges in the left frontal and central regions. A favorable prognosis was achieved in response to vitamin B6 and topiramate when the patient was seven months old. Our study expands the phenotype and genotype spectrum of FFEVF and provides solid diagnostic evidence for FFEVF.

Journal of Human Genetics (2022) 67:79-85; https://doi.org/10.1038/s10038-021-00969-z

\section{INTRODUCTION}

NPRL2 is a component of the GATOR1 complex, along with nitrogen permease regulator like-3 (NPRL3) and DEPDC5. The NPRL2 protein is expressed in variable regions of the human brain, including the frontal, temporal, parietal, and occipital lobes, similar to DEPDC5 [1]. NPRL2 is also known as tumor suppressor candidate 4 [2], which is upregulated in primary prostate cancer tissues [3]. The GATOR1 complex inhibits mechanistic target of rapamycin (mTOR) activation according to the amino acid levels in ambient cells [4]. It changes the nucleotide loading status (GTP or GDP) of the Rag proteins and deactivates them to release mTOR complex 1 (mTORC1) from the lysosome $[5,6]$.

mTORC1 has been described as a possible cause of epileptogenesis, and increased activity participates in seizure progression $[7,8]$. Overall, germline variants in the GATOR1 complex genes (DEPDC5, NPRL3, and NPRL2) are present in $~ 10 \%$ of focal epilepsy cases [9], which can be familial or sporadic, especially in familial focal epilepsy with variable foci (FFEVF). Moreover, generalized epilepsy and infantile spasms have also been reported [10]. Loss-of-function variants in DEPDC5 and $N P R L 3$ have been investigated extensively in both animal models and human tissues associated with mTORC1 hyperactivation $[11,12]$. However, variants in NPRL2 related to epileptogenesis are still not well understood.

Limited studies have suggested that variants of NPRL2 are correlated with focal epilepsy, which may be associated with focal cortical dysplasia (FCD) and intellectual disability $[1,10,13]$. Our study uncovered a new variation (NM_006545.5, c.339+2T>C) in NLPR2 in a 7-month-old infant with FFEVF that was inherited from his mother. The variation was confirmed to impact the alternative splicing of NPRL2, which may be the consequence of neurogenesis dysplasia through disturbing the mTOR signaling pathway. Our study expands the phenotype and genotype spectrum of NPRL2.

\section{MATERIALS AND METHODS \\ Patient}

Informed consent was obtained from the parents and their families. This study was approved by the institutional review board of the West China Second University Hospital. The patient's clinical manifestations, electroencephalogram (EEG), brain magnetic resonance imaging (MRI), malformations, investigations of other organs, and gene variations were analyzed. We also combined the NPRL2 variant-related cases reported previously in our analysis. Additional phenotype data and genetic findings for individuals are summarized in Table 1.

\begin{abstract}
Whole-exome sequencing (WES) and Sanger sequencing
To further clarify the patient's diagnosis, genomic DNA was extracted from the peripheral blood of the patient and his parents. WES was performed based on the NovaSeq 6000 Sequencing platform, IDT XGen Exome Research Panel was used to capture libraries, and paired-end clean reads were used to compare to the human reference genome (GRCh38/hg38). Variations were annotated through ANNOVAR [14] and picked up with a minor allele frequency of $\leq 0.005$ in the SNP database.

WES uncovered potential pathogenic variants. All variants were evaluated according to the American College of Medical Genetics and Genomics (ACMG) guidelines. "Ada" and "RF" scores were used to evaluate potential splicing variants predicted by dbscSNV. Sanger sequencing was performed to validate the variation identified by WES.
\end{abstract}

\footnotetext{
${ }^{1}$ Department of Pediatrics, West China Second University Hospital, Sichuan University, Chengdu, China. ${ }^{2}$ Key Laboratory of Obstetrics \& Gynecologic and Pediatric Diseases and Birth Defects of the Ministry of Education, Sichuan University, Chengdu, Sichuan, China. ${ }^{3}$ Cipher Gene LLC, Beijing, China. ${ }^{4}$ Department of Pediatrics, The City Central Hospital of Wanyuan, Wanyuan, Sichuan, China. ${ }^{\circledR}$ email: gordonrachel@scu.edu.cn
} 
1

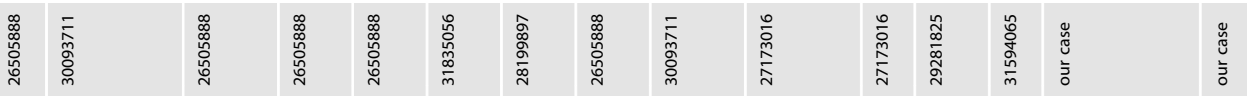

II

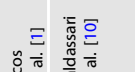

s言

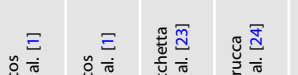

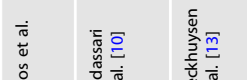

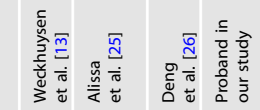

In

I:

Is

11

Is

Ill

乏)

Int

$\leqslant \frac{1}{1}$

$\div$ I

1

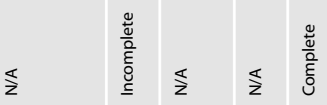

I

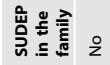

울요

1

1

I I

!

| I I I I I

I

$\|_{\text {I }}$

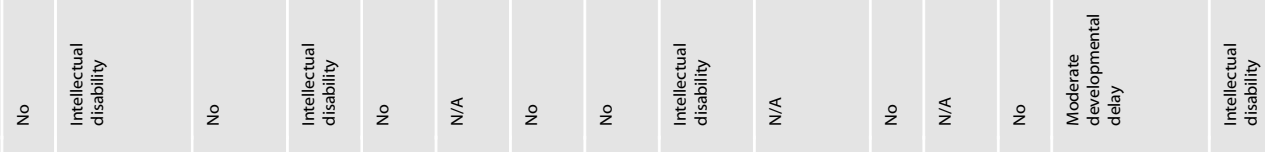

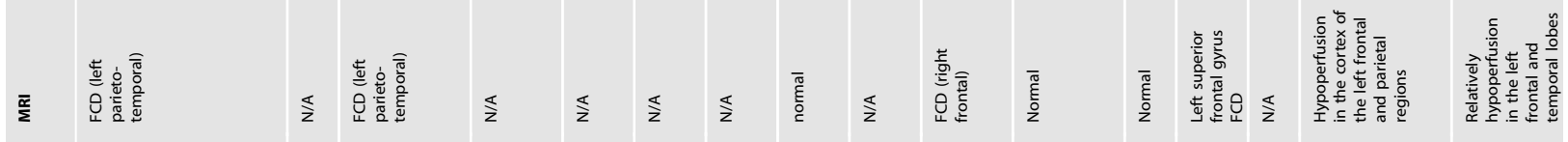

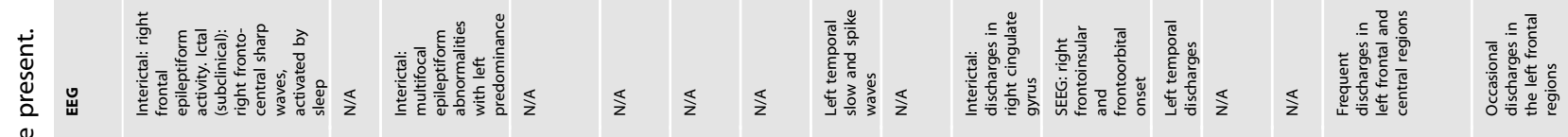

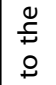

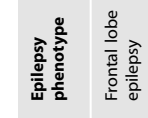

li!

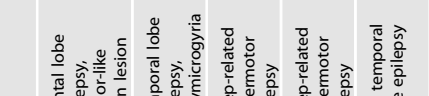

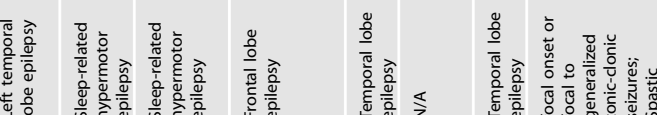

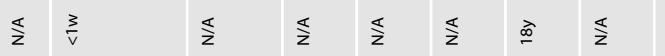

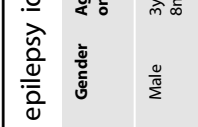

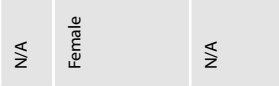

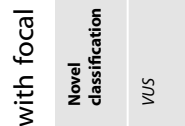

$\|$

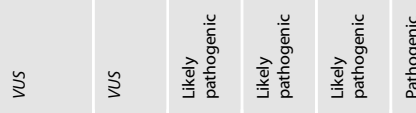

$\stackrel{\frac{a}{2}}{\frac{\pi}{\tilde{m}}}$

ते

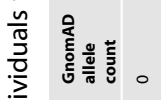

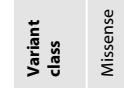

$\circ$

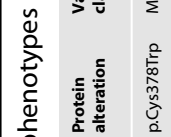

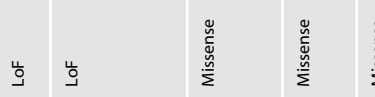

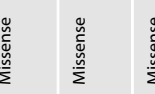

紊

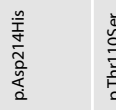

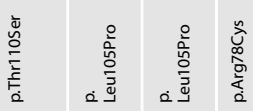

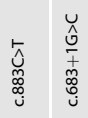

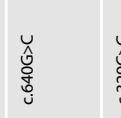

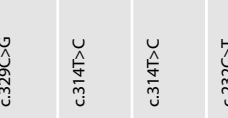

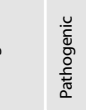

产

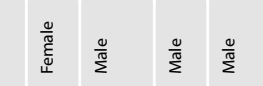

ते

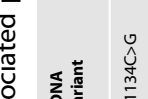

它

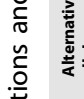

离

$\frac{\vec{r}}{\frac{1}{2}}$

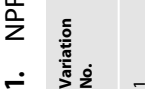

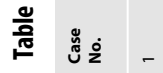




\section{Minigene construction}

Minigene assays were performed to investigate NPRL2 splicing via WT genomic DNA amplification. The construction contained exon 2-exon 3-exon 4 in NPRL2. Nested PCR was performed to amplify the targeted DNA fragment through normal peripheral blood. Amplification products were successfully cloned and confirmed by Sanger sequencing. Variation c.339+2T>C in NPRL2 was constructed by site-directed mutagenesis. Both wild-type and mutant fragments were delivered into the pCDNA3.1 vector after digestion and connection. All primers used in the minigene construct are provided in Supplementary Table 1.

\section{Cell transfection}

HeLa and 293 cells were cultured in DMEM supplemented with $10 \%$ fetal bovine serum. These minigenes, which are named pcDNA3.1-NPRL2-WT/ MUT, were transfected into 293T cells using Lipo2000 Transfection Reagent (11668019, 205 Invitrogen) according to the manufacturer's protocol. The DNA-lipid complex was incubated for $15 \mathrm{~min}$ in Opti MEM medium (Gibco, Grand Island, NY) at room temperature before addition to the cells.

\section{RT-PCR}

Total RNA was extracted $48 \mathrm{~h}$ after transfection using RNAiso PLUS (9109, TaKaRa). Retrotranscription was performed using a Prime Script RT Reagent kit with gDNA Eraser (RR047A, TaKaRa). Primers are shown in Supplementary Table 1. PCR was performed and evaluated on a $1 \%$ agarose gel. Subsequently, potential changes in the splicing process were identified by direct sequencing.

\section{D protein structure modeling}

Molecular modeling analysis was performed to estimate the variant in protein structure. WT and variations in NPRL2 protein were predicted using the Swiss-Model program. Swiss-Pdb Viewer software was used to visualize the structures between WT and variation proteins.

\section{RESULTS}

\section{Case presentation}

A 7-month-old male infant was born after an uneventful full-term pregnancy. He had multiple unprovoked seizures 4 days after birth, predominantly focal seizures or focal to generalized tonic-clonic seizures in semiology. The seizures occurred six times a day, most of which lasted $10 \mathrm{~s}$ and exhibited no diurnal differences. He developed spastic seizures when he was 1 month old. He was unable to roll over upon admission at 4 months of age with generalized hypotonia evident on the right side. There were no neurocutaneous markers, specific facial features, or any other systemic abnormalities. His occipitofrontal head circumference was $42.5 \mathrm{~cm}$. A diagnosis of developmental epileptic encephalopathy was considered. Interictal EEG demonstrated frequent sharp and slow waves in the left frontal and central regions (Supplementary Fig. 2a). Ictal EEG demonstrated the typical findings of epileptic spasms (Supplementary Fig. 2b-d). Brain CT, blood glucose, and electrolyte levels were normal. There were no abnormalities in the initial screening for infection or in the blood and urine metabolic screening. In addition, the auditory brainstem response and visual evoked potential tests were negative. His seizures were effectively controlled by a large dose of vitamin B6 $\left(50 \mathrm{mg} / \mathrm{kg} / \mathrm{d}^{*} 2\right.$ weeks) and topiramate $(8 \mathrm{mg} / \mathrm{kg} / \mathrm{d})$. Since the seizures free were acquired, the parents refused the treatment of adrenocorticotropic hormone. However, he still exhibited a failure to thrive, and his development was delayed and stagnant. At his latest follow-up at 7 months of age, he was seizure-free and sustained on a combination of topiramate $(8 \mathrm{mg} / \mathrm{kg} / \mathrm{d})$ and vitamin B6 (10 mg bid) therapy. He had moderate developmental delay, with a Gesell Developmental Scale score of 45 . He could lift his neck at 3 months of age and roll over at 5 months of age. However, he will not sit alone or take the initiative to grasp at 7 months old. Repeated EEG monitoring showed improvement in epileptiform discharge with intermittent sharp and slow waves in the left frontal and central regions. Head MRI showed no obvious signal abnormalities (Supplementary Fig. 1a-c). Interictal arterial spin labeling (ASL)-MRI showed relative hypoperfusion in the cortex of the left frontal and parietal regions (Supplementary Fig. 1g-j). His mother had an intellectual disability (WISC Scale score of 53). Her head MRI was normal (Supplementary Fig. 1d-f), while her ASL-MRI showed relative hypoperfusion in the left frontal and temporal lobes (Supplementary Fig. 1k-n). Her EEG showed occasional sharp and slow waves in the left frontal regions (Supplementary Fig. 2e, f). Both the patient's mother and grandfather had a history of febrile seizures in childhood.

\section{Identification of NPRL2 variation related to family focal epilepsy with variable foci}

WES was performed to further clarify the diagnosis and explore the etiology of our patient. A heterozygous variant (NM_006545.5: exon 3: $c .339+2 T>C$ ) in the NPRL2 gene was uncovered, and it was inherited from his mother (heterozygous) as determined through Sanger sequencing (Fig. 1a, b). The variant was a canonical splicing site in the $5^{\prime}$ end of intron 3 , which may impact alternative splicing.

Neither the GnomAD nor Exome Aggregation Consortium (ExAC) databases exhibited this variation, indicating the rarity of this mutation. It was also predicted to be splicing-influencing by "Ada" and "RF" scores (Table 2). Only 27 variants of NPRL2 were included in the ClinVar database (https://www.ncbi.nlm.nih.gov/ clinvar/?term $=$ NPRL2\%5Bgene\%5D), 16 of which were pathogenic or likely pathogenic. To date, 11 variants in the NPRL2 gene have been described in 14 epilepsy probands (Fig. 1c, Table 1). Fifty percent (7/14) of them are pathogenic, $21.4 \%$ (3/14) are likely pathogenic, and $28.6 \%$ (4/14) are variants of unknown significance (VUS). Five variants have been reported to have loss of function (LoF) and were classified as pathogenic in unrelated cases. The c.339+2T>C mutation in our study was also designated as likely pathogenic by the ACMG guidelines.

\section{Functional splicing examination of the variant through minigene assays}

Since the c.339+2T>C variant changed the $5^{\prime}$ donor site ' $g t^{\prime}$ in the intron region, a splicing assay was performed to further confirm the influence of alternative splicing. Target DNA fragments were successfully inserted into the pcDNA3.1 vector and confirmed by Sanger sequencing (Fig. 2a). RT-PCR results indicated two different splicing patterns, named "a" and " $c$ " and "b" and "d" (Fig. 2b). Sanger sequencing uncovered abnormal splicing in products " $b$ " and " $\mathrm{d}$ ". Intron 2 retention and exon 3 skipping of $18 \mathrm{bp}$ were observed in the mutant group (Fig. $2 c$, d). The schematic for abnormal splicing is shown in Fig. 2d. The red asterisk indicates the variation site. In addition, exon 3 in NPRL2 is highly conserved across multiple species (Fig. 2e).

\section{Protein modeling}

Swiss-Pdb Viewer was used to predict and compare the structures of NPRL2 in the WT and variant. Variation $339+2 T>C$ causes exon 3 skipping, which results in several amino acid stretches being deleted. Two sheets and one helix for NPRL2 in the N-terminal region disappeared compared with the WT (Fig. 3). These regions were highlighted with amino acid residues and van der Waals dots and will significantly affect the function and stability of the NPRL2 protein.

\section{DISCUSSION}

mTORC1 is well known to be involved in cell differentiation and protein synthesis and is highly expressed in the brain and regulates neurogenesis. Defects in the mTOR pathway play an important role in focal epilepsy during brain development. GATOR1, a negative regulator of $\mathrm{mTORC} 1$, consists of components DEPDC5, NPRL2, and NPRL3. In recent years, GATOR1-related 
(a)

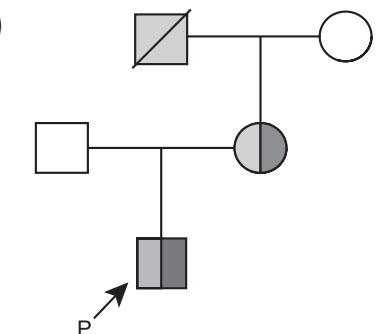

$\square$ Febrile Seizures $\square$ Intellectual Disability Spastic Seizures

Focal onset or focal to generalized tonic-clonic Seizures

(c)

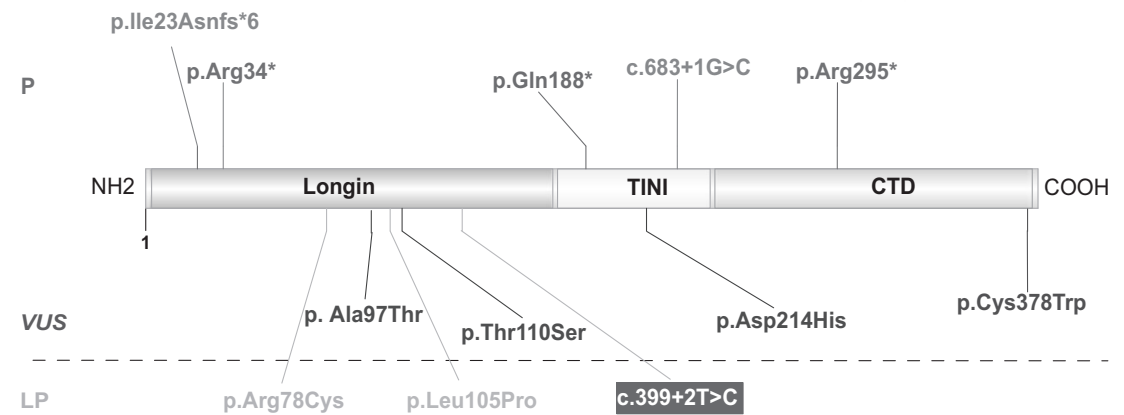

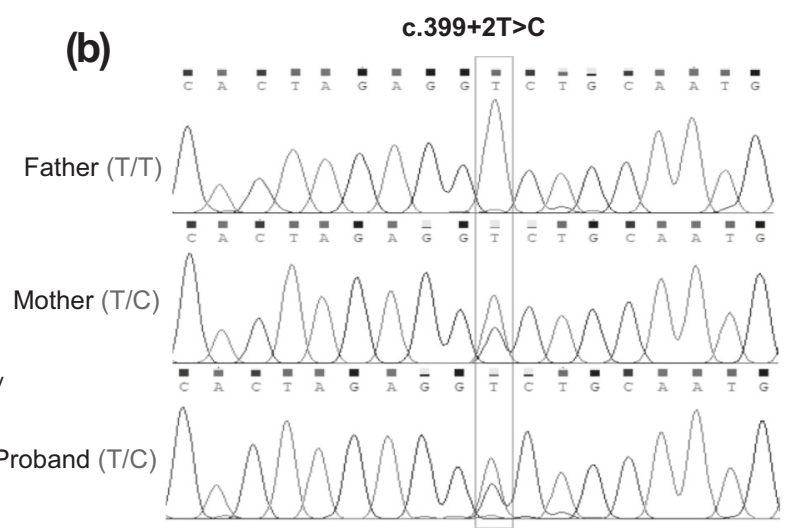

Fig. 1 Identification of a heterozygous variation in NPRL2. a Pedigree of the family. The proband affected with focal epilepsy is indicated by filled symbols with arrows. b Sanger sequencing of the proband and his parents showed a c.339+2T $>C$ mutation (red translucent box) in the NPRL2 gene, which was inherited from the mother. c Domain structure and modeling of NPRL2 variations in previous studies. The upper panel indicates the pathogenic (P) variants (red font). Variants of uncertain significance (VUS) and likely pathogenic (LP) are presented below. The NPRL2 protein contains an N-terminal longin domain (NLD), a tiny intermediary of NPRL2 that interacts with the DEPDC5 domain (TINI), and a C-terminal domain (CTD). The variation in our study in the red box was LP.

Table 2. Clinical examinations and variant information.

\begin{tabular}{|c|c|c|c|c|c|c|c|c|}
\hline \multirow[t]{2}{*}{ Gene } & \multirow[t]{2}{*}{ Mutation } & \multirow[t]{2}{*}{ Inheritance } & \multicolumn{3}{|l|}{ MAF } & \multirow{2}{*}{$\begin{array}{l}\text { dbscSNV } \\
\text { AD_SCORE }\end{array}$} & \multirow{2}{*}{$\begin{array}{l}\text { dbscSNV } \\
\text { RF_SCORE }\end{array}$} & \multirow[t]{2}{*}{ Category } \\
\hline & & & ExAc & GnomAD & 1000 Genome & & & \\
\hline NPRL2 & c. $339+2 \mathrm{~T}>\mathrm{C}$ & Inherited & NE & $\mathrm{NE}$ & $\mathrm{NE}$ & 0.9995 & 0.866 & LP \\
\hline
\end{tabular}

Transcript: NM 006545.5.

$M A F$ minor allele frequency, $N E$ not exist, $L P$ likely pathogenic.

variations have been gradually recognized in epilepsy patients via the wide application of second-generation sequencing, causing $\sim 10 \%$ of focal epilepsy $[1,13]$. GATOR 1 variations that activate the mTORC1 pathway assume the main responsibility for focal epilepsy with cortical malformations, representing a potential target for novel therapeutics [10].

Familial focal epilepsy (FFE) is characterized as a genetically distinct type of epilepsy syndrome, primarily including autosomal dominant nocturnal frontal lobe epilepsy, familial medial temporal lobe epilepsy, familial lateral temporal lobe epilepsy, and FFEVF, which are considered to primarily result from ion channel and neurotransmitter receptor gene variations. FFEVF is a typical subtype that has been reported to be primarily associated with DEPDC5 variations. At the same time, $\sim 12 \%$ of FFE patients have been found possess DEPDC5 variations [15]. Epilepsy syndrome caused by NPRL2 and NPLR3 variations is similar to DEPDC5 [1]. EEG showed that the initial discharge site was changeable, mostly from the frontal lobe or temporal lobe. However, no obvious correlation between EEG discharge and clinical manifestations was found [16]. GATOR1-related FFEVF patients always have a family history, with incomplete penetrance and heterogeneous clinical manifestations. Some of them exhibit focal EEG discharge without clinical symptoms.
Most patients present with normal psychomotor development, while some may have mild cognitive decline or autism-like manifestations without seizures [1, 15]. Similarly, our case presented multiple seizure types, including infantile spasms, focal seizures, or focal to generalized tonic-clonic seizures. Both his mother and grandfather had a history of febrile seizures when they were young. In addition, his mother had an intellectual disability.

Approximately $20 \%$ of patients with GATOR1-related focal epilepsy have FCD $[10,13,16]$. In our study, no obvious abnormalities were observed in MRI of this proband. ASL-MRI showed relative hypoperfusion in the left frontal and parietal lobes, consistent with the epileptiform discharge detected by video EEG. It has been reported that $55-78 \%$ of GATOR1-related focal epilepsy patients are drug-resistant $[10,17]$. Patients with definite lesions had better surgical results, with a $50-60 \%$ complete remission rate $[10,18]$. Fortunately, our case had a favorable prognosis with the combination treatment of topiramate and vitamin B6, and the video EEG on follow-up showed a significant decrease in epileptiform discharges. Considering the focal seizures with epileptic spasms of the proband, high-dose vitamin B6 and topiramate were applied according to the guidelines and consensus on the treatment of infantile spasms 
(a)

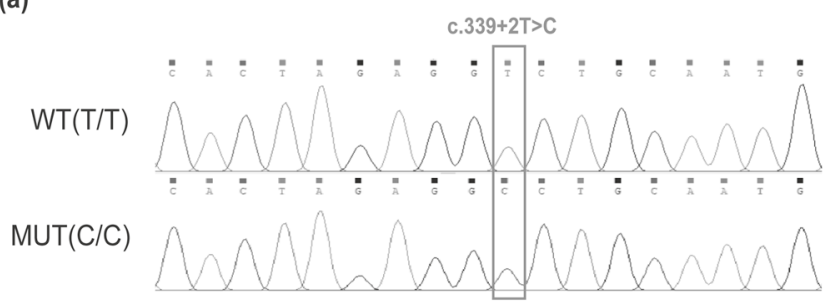

(c)

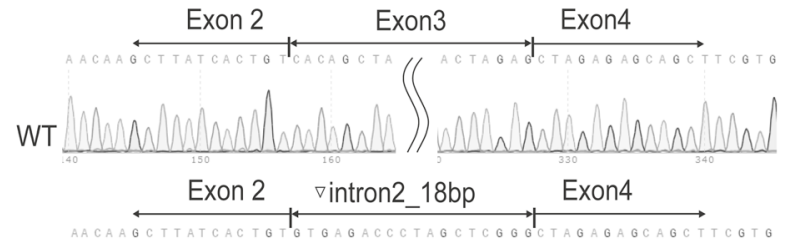

MUT

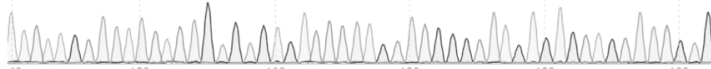

(b)

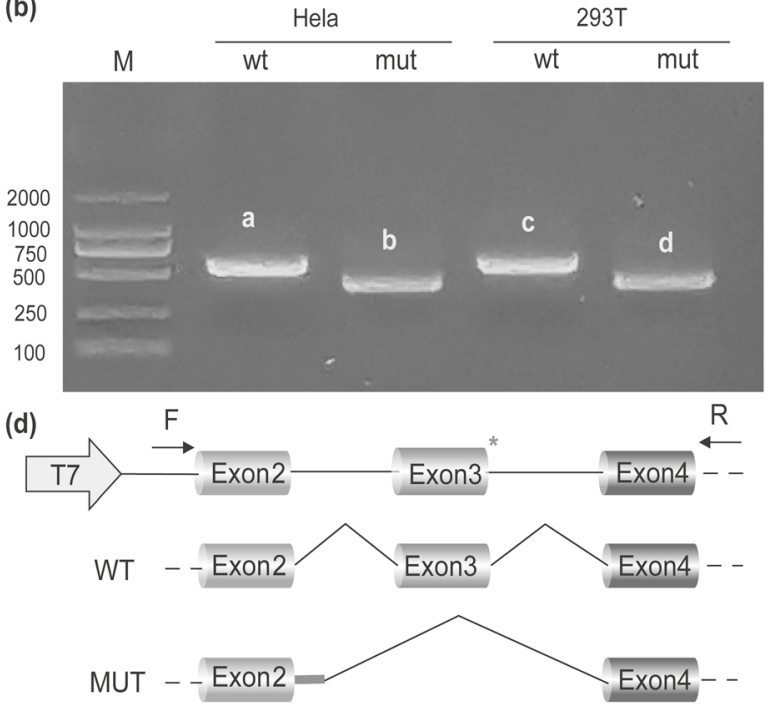

Exon3

(e)

Homo sappiens QNKL I TVTAMEKKL IGCPVCIEHKKYSRNALLFNLGFVCDAQAKTCALEP IVKKLAGYLTTLELES SFVSMEE Mus_musculus QNKL I TVTAMEKKLIGCPVC IEHKKYSRNALLFNLGFVCDAQAKTCALEP IVKKLAGYLTTLELE S S FVS SEE Rattus_ñnorvegicus QNKL I TVTAMEKKL IGCPVC IEHKKYSRNALLFNLGFVCDAQAKTCALEP IVKKLAGYLTTLELES S FVS TEE

Danio rerio QNKL I TVTAMEKKL I GCPVC IEHKKYRRNALLFNLGLVCDASTKTSALEP IVKKLAGYLTTLELE S GF I S NEE Gallus_gallus QNKL I TVTAMEKKL I GCPVC IEHKKYSRNALLFNLGFVCDARAKACALEP IVKKLAGYLTTLELESGF I S NKE Oryctolagus_cuniculus QNKL I TVTAMEKKL IGCPVCIEHKKYSRNALLFNLGFVCDAQATTCALEPIVKKLAGYLTTLELES S FV S TEE

Fig. 2 Minigene assays and identification of the variant's impact on alternative splicing. a Sanger sequencing confirmed that wild-type and mutant fragments were successfully introduced into the minigene construct. Splicing variation c.339+2T $>C$ in NPRL2 is indicated by the red box. b RT-PCR was performed to verify alternative splicing in the wild-type and mutant groups. Abnormal splicing bands in mutant groups, named " $b$ " and " $d$ ", were uncovered in both HeLa and 293 T cells. At the same time, normal bands, named "a" and " $c$ ", were indicated in the wild-type group. c Alternative splicing was affected by the c.339+2T>C variation in NPRL2. PCR product sequencing revealed 18 bp intron 2 retention and exon 3 skipping. The alternative schematic is shown in (d). The red "*" symbolizes the variation site. Intron 2 retention is indicated in the mutant group by a red line. e Species conservation analysis of exon 3 in NPRL2.
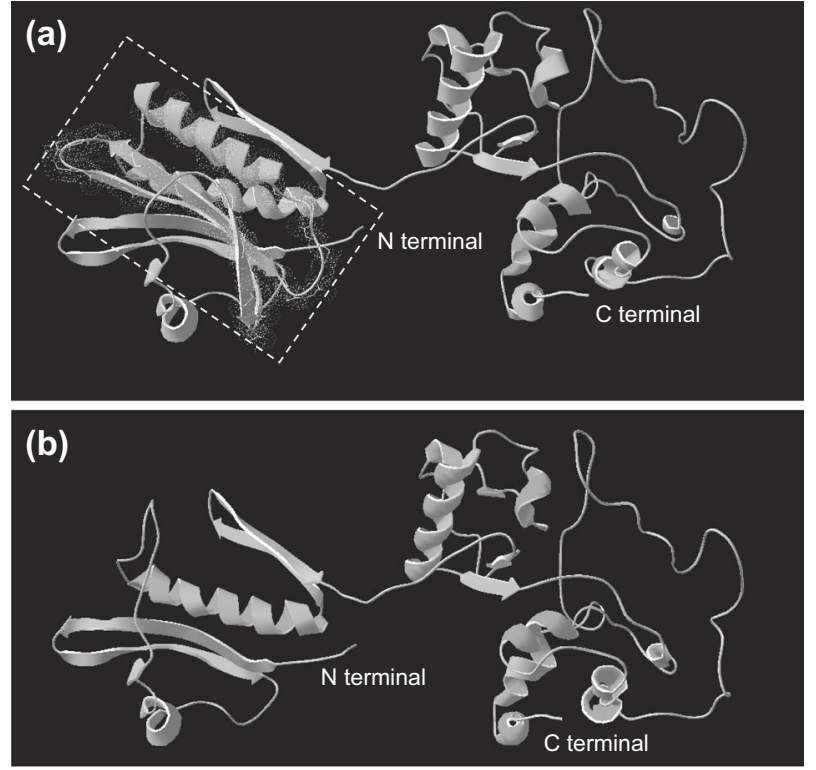

Fig. 3 Protein 3D structures in WT and the NPRL2 variant. a WT protein. b Variant protein. The yellow dotted box indicates the different regions of the WT NPRL2 protein compared to the variation.

and epileptic encephalopathy [19]. However, there are not any apparent relationships between the NPRL2 gene and the mechanisms of action of anti-seizure medications which needs more investigation in the future.
There is no clear correlation between genotype and phenotype in GATOR1 variations, with an incomplete penetrance from $50 \%$ to $82 \%[1,15]$. This likely results from a combination of genetic, environmental, and lifestyle factors [20]. Additionally, this may be due to the additive effect of multiple independent variations, which can disturb and often increase the severity of the phenotype. GATOR1-related epilepsies caused by DEPDC5, NPRL2, and NPRL3 variations exhibit various phenotypes consisting of sleep-related focal hypermotor seizures, infantile spasms, and other focal epilepsies, including frontal, temporal, occipital, parietal, centrotemporal epilepsies [10]. The various phenotypes are the same as those of patients who have NPRL2 variations. Previous studies also revealed distinct phenotypes in a family with the same variant $[1,13]$. A single pathogenic variant could lead to variable phenotypic manifestations, including different age at onset, seizure type, seizure severity, drug response, and presence of cortical malformations [16], which was observed in our comparison (Table 1). The types of variations seem to have no connection with the epilepsy phenotypes, neuropsychiatric comorbidities, or abnormal brain structure. Interfamilial variability in genetic epilepsies is a common phenomenon. Therefore, the child presented with developmental epileptic encephalopathy with focal as well as spastic seizures, while the mother showed only mild mental retardation. The maternal grandfather had a history of febrile seizures at a young age, and then had no epileptic seizures, and the psychomotor development was normal. One explanation for how a single variant in the same family causes mild focal epilepsy or refractory epilepsy may be the occurrence of a second somatic mutation [18].

Haploinsufficiency has been shown to be the pathogenic mechanism in NPRL2/3 variations with incomplete penetrance [13]. However, whether missense and splicing variations have 
clinical significance is still unclear. Functional evidence and strong segregation support were absent. Therefore, no missense or splicing variations were classified as pathogenic through the new framework of epilepsy-related GATOR1 classification [10]. Alternative splicing was confirmed by minigene assays, and exon 3 skipping was observed during transcription (Fig. 2). The mutation may destroy the original donor site in exon 3 and activate a cryptic splice site at the beginning of intron 2 [21, 22]. In addition, it should be mentioned that NPRL2 links DEPDC5 and NPRL3 to comprise the GATOR1 complex. Furthermore, NPRL2 interacts with DEPDC5 through its longin domain [6]. Variation in our study leads to exon 3 skipping located in the longin domain, which may affect the connection between NPRL2 and DEPDC5 and is critical for the function of the GATOR1 complex.

In summary, we describe an individual with multiple seizure types harboring a splicing variation in NPRL2. Exon 3 skipping was confirmed based on minigene assays, which may lead to a loss of function in NPRL2. Our study provides evidence for pathogenicity of the splicing variation in the GATOR1 complex and expands the phenotype and genotype spectrum of FFEVF, highlighting the critical role for NPRL2 in neurodevelopment.

\section{REFERENCES}

1. Ricos MG, Hodgson BL, Pippucci T, Saidin A, Ong YS, Heron SE, et al. Mutations in the mammalian target of rapamycin pathway regulators NPRL2 and NPRL3 cause focal epilepsy. Ann Neurol. 2016;79:120-31.

2. Lerman MI, Minna JD. The 630-kb lung cancer homozygous deletion region on human chromosome 3p21.3: identification and evaluation of the resident candidate tumor suppressor genes. The International Lung Cancer Chromosome 3p21.3 Tumor Suppressor Gene Consortium. Cancer Res. 2000;60:6116-33.

3. Chen Z, Jiang $Q$, Zhu P, Chen Y, Xie X, Du Z, et al. NPRL2 enhances autophagy and the resistance to Everolimus in castration-resistant prostate cancer. Prostate. 2019;79:44-53.

4. Bar-Peled L, Chantranupong L, Cherniack AD, Chen WW, Ottina KA, Grabiner BC, et al. A tumor suppressor complex with GAP activity for the Rag GTPases that signal amino acid sufficiency to mTORC1. Science. 2013;340:1100-6.

5. Nicastro R, Sardu A, Panchaud N, De Virgilio C. The architecture of the Rag GTPase signaling network. Biomolecules. 2017;7:48-69.

6. Shen K, Huang RK, Brignole EJ, Condon KJ, Valenstein ML, Chantranupong L, et al. Architecture of the human GATOR1 and GATOR1-Rag GTPases complexes. Nature. 2018;556:64-9.

7. Lasarge $\mathrm{CL}$, Danzer SC. Mechanisms regulating neuronal excitability and seizure development following mTOR pathway hyperactivation. Front Mol Neurosci. 2014;7:18.

8. Wong M. A critical review of mTOR inhibitors and epilepsy: from basic science to clinical trials. Expert Rev Neurother. 2013;13:657-69.

9. Baldassari S, Licchetta L, Tinuper P, Bisulli F, Pippucci T. GATOR1 complex: the common genetic actor in focal epilepsies. J Med Genet. 2016;53:503-10.

10. Baldassari S, Picard F, Verbeek NE, van Kempen M, Brilstra EH, Lesca G, et al. The landscape of epilepsy-related GATOR1 variants. Genet Med. 2019;21:398-408.

11. Scerri T, Riseley JR, Gillies G, Pope K, Burgess R, Mandelstam SA, et al. Familial cortical dysplasia type IIA caused by a germline mutation in DEPDC5. Ann Clin Transl Neurol. 2015;2:575-80.

12. Sim JC, Scerri T, Fanjul-Fernández M, Riseley JR, Gillies G, Pope K, et al. Familial cortical dysplasia caused by mutation in the mammalian target of rapamycin regulator NPRL3. Ann Neurol. 2016;79:132-7.

13. Weckhuysen S, Marsan E, Lambrecq V, Marchal C, Morin-Brureau M, AnGourfinkel I, et al. Involvement of GATOR complex genes in familial focal epilepsies and focal cortical dysplasia. Epilepsia 2016;57:994-1003.

14. McKenna A, Hanna M, Banks E, Sivachenko A, Cibulskis K, Kernytsky A, et al. The Genome Analysis Toolkit: a MapReduce framework for analyzing next-generation DNA sequencing data. Genome Res. 2010;20:1297-303.

15. Dibbens LM, de Vries B, Donatello S, Heron SE, Hodgson BL, Chintawar S, et al. Mutations in DEPDC5 cause familial focal epilepsy with variable foci. Nat Genet. 2013;45:546-51.

16. Baulac S. mTOR signaling pathway genes in focal epilepsies. Prog Brain Res. 2016;226:61-79.

17. van Kranenburg $M$, Hoogeveen-Westerveld $M$, Nellist $M$. Preliminary functional assessment and classification of DEPDC5 variants associated with focal epilepsy. Hum Mutat. 2015;36:200-9.
18. Baulac S, Ishida S, Marsan E, Miquel C, Biraben A, Nguyen DK, et al. Familial focal epilepsy with focal cortical dysplasia due to DEPDC5 mutations. Ann Neurol. 2015;77:675-83.

19. Wilmshurst JM, Gaillard WD, Vinayan KP, Tsuchida TN, Plouin $P$, Van Bogaert $P$, et al. Summary of recommendations for the management of infantile seizures: Task Force Report for the ILAE Commission of Pediatrics. Epilepsia. 2015;56:1185-97.

20. Coll M, Pérez-Serra A, Mates J, Del Olmo B, Puigmulé $M$, Fernandez-Falgueras $A$, et al. Incomplete penetrance and variable expressivity: hallmarks in channelopathies associated with sudden cardiac death. Biology. 2017;7:3-13.

21. Ham KA, Aung-Htut MT, Fletcher S, Wilton SD. Nonsequential splicing events alter antisense-mediated exon skipping outcome in COL7A1. Int J Mol Sci. 2020;21:7705-20.

22. Zhou K, Huang L, Feng M, Li X, Zhao Y, Liu F, et al. A novel SLC26A4 splicing mutation identified in two deaf Chinese twin sisters with enlarged vestibular aqueducts. Mol Genet Genom Med. 2020;8:e1447.

23. Licchetta L, Pippucci T, Baldassari S, Minardi R, Provini F, Mostacci B, et al. Sleeprelated hypermotor epilepsy (SHE): Contribution of known genes in 103 patients. Seizure. 2020;74:60-4.

24. Perucca P, Scheffer IE, Harvey AS, James PA, Lunke S, Thorne N, et al. Real-world utility of whole exome sequencing with targeted gene analysis for focal epilepsy. Epilepsy Res. 2017;131:1-8.

25. D'Gama AM, Woodworth MB, Hossain AA, Bizzotto S, Hatem NE, LaCoursiere CM, et al. Somatic Mutations Activating the mTOR Pathway in Dorsal Telencephalic Progenitors Cause a Continuum of Cortical Dysplasias. Cell Rep. 2017;21:3754-66.

26. Deng J, Fang F, Wang XH, Dai LF, Tian XJ, Chen $\mathrm{CH}$. [Clinical and genetic characteristics of focal epilepsy in children caused by GATOR1 complex gene variation]. Zhonghua Er Ke Za Zhi. 2019;57:780-5.

\section{ACKNOWLEDGEMENTS}

We would like to thank the proband and his parents for their kind cooperation and Cipher Gene LCC for sequencing.

\section{AUTHOR CONTRIBUTIONS}

JZ: Conceptualization, methodology, writing-original draft preparation; YS, ZY, FY: Writing-original draft preparation, software, data mining; YL, BY, WC: data mining, investigation; JG: supervision, writing-reviewing, and editing.

\section{FUNDING}

This work was supported by the National Science Foundation of China (Nos. 82071686 and 81501301), the Grant from Science and Technology Bureau of Sichuan province (No. 2021YFS0093), and the Grant from Clinical Research Fund of West China Second University Hospital (No. KL072), and the Grant of "Yun" Epilepsy Genetics Research and Applicant Fund.

\section{COMPETING INTERESTS}

The authors declare no competing interests.

\section{ETHICAL APPROVAL AND CONSENT TO PARTICIPATE}

Written informed consent was obtained from the patient's legal guardians to participate in this study. This study was approved by the human ethics committees of West China Second University Hospital.

\section{ADDITIONAL INFORMATION}

Supplementary information The online version contains supplementary material available at https://doi.org/10.1038/s10038-021-00969-z.

Correspondence and requests for materials should be addressed to J.G.

Reprints and permission information is available at http://www.nature.com/ reprints

Publisher's note Springer Nature remains neutral with regard to jurisdictional claims in published maps and institutional affiliations. 
Open Access This article is licensed under a Creative Commons Ay Attribution 4.0 International License, which permits use, sharing,
adaptation, distribution and reproduction in any medium or format, as long as you give appropriate credit to the original author(s) and the source, provide a link to the Creative Commons license, and indicate if changes were made. The images or other third party material in this article are included in the article's Creative Commons license, unless indicated otherwise in a credit line to the material. If material is not included in the article's Creative Commons license and your intended use is not permitted by statutory regulation or exceeds the permitted use, you will need to obtain permission directly from the copyright holder. To view a copy of this license, visit http://creativecommons. org/licenses/by/4.0/.

(c) The Author(s) 2021 